\title{
Characterization of speed fluctuation and drag force in young swimmers: A gender comparison
}

\author{
Tiago M. Barbosa ${ }^{\mathrm{a}, \mathrm{f}, *}$, Mário J. Costa ${ }^{\mathrm{b}, \mathrm{f}}$, Jorge E. Morais ${ }^{\mathrm{b}, \mathrm{f}}$, Pedro Morouço ${ }^{\mathrm{c}, \mathrm{f}}$, \\ Marc Moreira ${ }^{\mathrm{d}, \mathrm{f}}$, Nuno D. Garrido ${ }^{\mathrm{d}, \mathrm{f}}$, Daniel A. Marinho ${ }^{\mathrm{e}, \mathrm{f}}$, António J. Silva ${ }^{\mathrm{d}, \mathrm{f}}$ \\ a National Institute of Education, Nanyang Technological University, Singapore \\ ${ }^{\mathrm{b}}$ Polytechnic Institute of Bragança, Bragança, Portugal \\ ${ }^{c}$ Polytechnic Institute of Leiria, Leiria, Portugal \\ ${ }^{\mathrm{d}}$ University of Trás-os-Montes and Alto Douro, Vila Real, Portugal \\ ${ }^{\mathrm{e}}$ University of Beira Interior, Covilhã, Portugal \\ ${ }^{\mathrm{f}}$ Research Centre in Sports, Health and Human Development, Vila Real, Portugal
}

\section{A R T I C L E I N F O}

\section{Article history:}

Available online 24 September 2013

\section{PsycINFO classification:}

3720

Keywords:

Front crawl

Hydrodynamics

Active drag

Passive drag

Kinematics

Children

\begin{abstract}
A B S T R A C T
The aim of this study was to compare the speed fluctuation and the drag force in young swimmers between genders. Twenty-three young pubertal swimmers (12 boys and 11 girls) volunteered as subjects. Speed fluctuation was measured using a kinematical mechanical method (i.e., speedo-meter) during a maximal $25-\mathrm{m}$ front crawl bout. Active drag, active drag coefficient and power needed to overcome drag were measured with the velocity perturbation method for another two maximal $25 \mathrm{~m}$ front crawl bouts with and without the perturbation device. Passive drag and the passive drag coefficient were estimated using the gliding decay velocity method after a maximal push-off from the wall while being fully immersed. The technique drag index was also assessed as a ratio between active and passive drag. Boys presented meaningfully higher speed fluctuation, active drag, power needed to overcome drag and technique drag index than the girls. There were no significant gender differences for active drag coefficient, passive drag and passive drag coefficient. There were positive and moderate-strong associations between active drag and speed fluctuation when controlling the effects of swim velocity. So, increasing speed fluctuation leads to higher drag force values and those are even higher for boys than for girls.
\end{abstract}

(C) 2012 Elsevier B.V. All rights reserved.

\footnotetext{
* Corresponding author at: Physical Education \& Sports Science Academic Group, National Institute of Education, Nanyang Technological University. Singapore 637616. Tel.: +65 6219 6213; fax: +65 68969260.

E-mail address: tiago.barbosa@nie.edu.sg (T.M. Barbosa).
} 


\section{Introduction}

Swimming is an aquatic locomotion technique based on periodic limb actions to overcome drag force and propel the body forward in the water. The subject's arms, legs and trunk actions within a swimming stroke cycle lead to changes in the velocity described as:

$$
v=v_{0}+\Delta v(t)
$$

where $v$ is the swimmer's mean velocity, $v_{0}$ is the swimmer's velocity at the beginning of the stroke cycle, $\Delta v$ is the variation of the swimming velocity throughout the stroke cycle and $t$ is the time (Barbosa, Bragada et al., 2010). In this sense, the swimmer is not able to sustain a uniform movement (i.e., $\Delta v=0 \mathrm{~m} / \mathrm{s}$ ). Instead, he/she is submitted to an intra-cyclic variation of the horizontal velocity of his/ her body, also known as 'speed fluctuation' (i.e., $\Delta v \neq 0 \mathrm{~m} / \mathrm{s}$ ).

The speed fluctuation, considering a given period of time, defines the swimmer's acceleration and is dependent on the applied resultant force, as well as the inertial term of Newton's equation of motion:

$$
F=m \cdot a
$$

where $F$ is the resultant force, $m$ is the body mass and $a$ is the acceleration. In competitive swimming (i) the resultant force is the balance between propulsion and drag; (ii) the inertial term includes the swimmer's body mass plus the added water mass, and (iii) the body's acceleration (Seifert, Toussaint, Alberty, Schnitzler, \& Chollet, 2010; Vilas-Boas et al., 2010):

$$
\operatorname{Pr}+D=\left(B M+m_{a}\right) \cdot a
$$

where $P r$ is the total of all propulsive forces, $D$ is the drag force, $B M$ is the swimmer's body mass, $m_{a}$ is the added water mass and $a$ is the swimmer's acceleration.

Theoretically there seems to exist a relationship between the swimmer's hydrodynamic profile and his or her swimming kinematics. Indeed, few research attempts have been made to uncover the relationship, or co-variance, between speed fluctuation and drag force (e.g., Schnitzler, Seifert, Ernwein, \& Chollet, 2008; Seifert et al., 2008). Drag force can be assessed (i) with the swimmer being towed or gliding in the hydrodynamic position, without any further limb action - passive drag, or (ii) with the swimmer performing limb action to propel him/herself forward in the water - active drag. Both passive and active drag can be measured using numerical simulations as well as experimental methods (Marinho et al., 2009). Several experimental methods have been reported in literature to measure passive and active drag. Passive drag can be measured with the gliding decay velocity method (Klauck \& Daniel, 1976). In this method, it is assumed that the ratio of velocity decay gliding in the hydrodynamic position, after a push-off from the wall, can estimate the drag force to which the swimmer is submitted. For the measurement of active drag, the velocity perturbation method might be used (Kolmogorov \& Duplischeva, 1992). This method assumes that the power output to overcome drag is maximal and constant while swimming with and without a perturbation device attached to the swimmer. Active drag can be calculated since power to drag equals drag force times speed.

The ratio of active drag to passive drag is one of the main concerns for swimming researchers. It seems that there is no consistent evidence about the exact difference between passive and active drag intensities. Some authors suggested that active drag is: (i) almost twice the value of passive drag measured with the $\mathrm{VO}_{2}$ back-extrapolation method (di Prampero, Pendergast, Wilson, \& Rennie, 1974; Zamparo, Gatta, Pendergast, \& Capelli, 2009); (ii) ranging between 0.5 and 1.5 times the passive drag using the velocity perturbation method in adult swimmers (Kjendlie \& Stallman, 2008; Kolmogorov \& Duplischeva, 1992); (iii) being almost the same value of the passive drag using the measuring active drag method in adult swimmers (Toussaint et al., 1988; van der Vaart et al., 1987) and young swimmers with the velocity perturbation method (Kjendlie \& Stallman, 2008). Data variations among studies might be related to differences in the methods applied to assess both passive and active drag, as well as, the competitive level, age and gender of the subjects evaluated. This ratio of active to passive drag was widely broadcasted in literature after having been reported by Kolmogorov and Duplischeva (1992). Thereafter, Kjendlie and Stallman (2008) designated the active-passive drag ratio as 'tech- 
nique drag index' (TDI) and considered it as one more swimming efficiency index. The rationality behind TDI, as a swimming efficiency index, is that if two swimmers with similar passive drag are compared, the one with lower active drag could be considered as having a better technique (Kjendlie \& Stallman, 2008). It is considered that lower-level swimmers will have an increased TDI in comparison to high-level ones as a result of a lower efficiency.

The literature describing the drag force in young swimmers is scarce. As far as we know, there is no study regarding speed fluctuation in children or comparing both drag force and speed fluctuation between boys and girls. At least two studies compared the change in speed fluctuation according to gender in adult swimmers. In general, adult female swimmers have a lower speed fluctuation than their male counter partners in breaststroke (Manley \& Atha, 1992) and front crawl (Schnitzler et al., 2008). Those differences can be related to differences in anthropometric properties and mechanical power output. As can be appreciated from Eqs. (2) and (3), body mass (anthropometrics) and acceleration (mechanical power) have an influence on propulsion and drag forces. When compared to adult males, the adult females had a lower mechanical power output and a lower drag force to overcome, which accounts for the lower speed fluctuation (Schnitzler et al., 2008).

It is uncertain if gender differences in drag force and speed fluctuation during childhood/puberty are similar to the ones described during adulthood. Regarding the kineanthropometric profile, literature suggests there are no significant differences between boys and girls until puberty (Malina, Bouchard, \& Bar-Or, 2004). However, at the start of puberty a gender gap exists, also for competitive swimmers (Seifert, Barbosa, \& Kjendlie, 2010). Peri-pubertal subjects have greater morphometric characteristics (e.g., total and partial body lengths, body mass, body areas and body volumes) than pre-pubertal ones. In addition, throughout and up to the end of puberty males become taller and heavier than females. There is evidence that greater anthropometric characteristics lead to a higher resistance in aquatic locomotion (i.e., passive and active drag). Regarding the swimming technique, several variables have been selected on a regular basis to assess a swimmer's 'overall' technique. As for the anthropometrics, by the end of puberty motor control and inter-limb coordination may play a prominent role in swimming technique enhancement. Anthropometrics is also a determinant of swimming technique. For instance, throughout puberty arm span increases and so do the stroke cycle and the swimming velocity as a consequence of that. It follows from Eq. (3) that one of the most feasible variables to assess 'overall' swimming technique is speed fluctuation (Vilas-Boas, Fernandes, \& Barbosa, 2011). If a gender difference exists in drag force and/or speed fluctuation at a given moment of the swimmer's career (from childhood to adulthood, i.e., during puberty) it might be related to some or all of these factors. At some point in puberty boys reach the biological maturation of the girls and become taller, heavier, with more muscle strength, as well as presenting a better motor control and coordination (i.e., technique).

Therefore, the aim of this study was to compare the speed fluctuation and the drag force in young swimmers according to gender. It was hypothesized that, as in adult swimmers, boys will present a higher speed fluctuation and drag force compared to girls.

\section{Methods}

\subsection{Subjects}

Twenty-three young swimmers (12 boys and 11 girls) who participated on a regular basis in regional and national level competitions volunteered as subjects. The cohort groups (boys versus girls) were split according to biological/sex maturation. Boys and girls were approximately at the 3rd stage of the Tanner scale (boys: $3.33 \pm 0.78$ Tanner stages by self-evaluation; $14.42 \pm 1.24$ years old, $1.66 \pm 0.09 \mathrm{~m}$ of height, $56.45 \pm 10.80 \mathrm{~kg}$ of body mass; girls: $3.00 \pm 0.89$ Tanner stages by self-evaluation; $12.73 \pm 0.79$ years old, $1.60 \pm 0.05 \mathrm{~m}$ of height, $47.55 \pm 6.27 \mathrm{~kg}$ of body mass).

Parents and coaches gave their consent for the swimmers' participation in this study. All procedures were in accordance to the Declaration of Helsinki with respect to human research. The Institutional Review Board of the Polytechnic Institute of Bragança approved the study design. 


\subsection{Kinematics mechanical method}

Each swimmer made a maximal $25-\mathrm{m}$ front crawl swim with an underwater start. Subjects performed the bout alone with no other swimmer in the lane or nearby lanes to reduce drafting and pacing effects, affecting the drag force. The swimmers were advised to reduce gliding during the start.

A speedo-meter cable (Swim speedo-meter, Swimsportec, Hildesheim, Germany) was attached to the swimmer's hip. Bio-signal was acquired on-line at a sampling rate of $50 \mathrm{~Hz}$. The speedo-meter was placed in the forehead-wall of the swimming pool, about $0.2 \mathrm{~m}$ above the water surface. A software's interface in LabVIEW ${ }^{\circledR}$ (v. 2009) was used to acquire, display and process pair-wise velocitytime data on-line during the swim bout. To transfer data from the speedo-meter to the software application, a 12-bit resolution acquisition card (USB-6008, National Instruments, Austin, Texas, USA) was used as well. The integrated system (hardware plus software) was validated by means of Doppler and videometric methods, respectively (Barbosa, Costa et al., 2011a, 2011b). Age-group coaches and researchers are aware that weak kicking is a major issue in young swimmers. Although there is no solid scientific evidence for that, it was assumed that the absolute contributions of kicking to total velocity might be even lower in children than in adult swimmers ( 10-15\%). Therefore, the turbulent action of the water inducing $d v$ 's bias might be almost negligible for these young swimmers. During data collection an evaluator visually inspected the curves being drawn in the software's interface while the swimmer performed the bout. If some technical/methodological issue occurred (e.g., bumping feet on the cable), evaluators asked the swimmers to repeat the bout in question.

Thereafter, data were exported to signal processing software (AcqKnowledge v. 3.5, Biopac Systems, Santa Barbara, USA) and filtered with a $5 \mathrm{~Hz}$ cut-off low-pass 4th order Butterworth filter. The intra-cyclic variation of the horizontal velocity of the hip $(d v)$ was analyzed as described by Barbosa, Bragada et al. (2010):

$$
d v=\frac{\sqrt{\frac{\sum_{i}\left(v_{i}-\bar{v}\right)^{2} \cdot F_{i}}{n}}}{\frac{\sum_{i} v_{i} \cdot F_{i}}{n}} \cdot 100
$$

where $d v$ represents the intra-cyclic variation of the horizontal velocity of the hip, $v$ represents the mean swimming velocity, $v_{i}$ represents the instant swimming velocity, $F_{i}$ represents the acquisition frequency, and $n$ is the number of observations. For further analysis the $d v$ mean value of three consecutive stroke cycles between the 11th $\mathrm{m}$ and 24 th $\mathrm{m}$ from the starting wall was considered.

\subsection{Velocity perturbation method}

The velocity perturbation method was used to determine active drag in front crawl swimming with the help of an additional hydrodynamic body (Kolmogorov \& Duplischeva, 1992; Kolmogorov, Rumyantseva, Gordon, \& Cappaert, 1997). Active drag was calculated from the difference between the swimming velocities with and without towing the perturbation buoy. To ensure similar maximal power output for the two sprints, the swimmers were instructed to perform maximally at both $25 \mathrm{~m}$ trials. Between bouts swimmers rested for at least $30 \mathrm{~min}$. Each swimmer performed two maximal $25 \mathrm{~m}$ front crawl bouts with an underwater start with and without the perturbation device. Subjects performed the bouts alone without any other swimmer in the same swim lane and in the nearby lanes to reduce drafting, pacing effects and bias in the drag force. Swimming velocity was assessed during $13 \mathrm{~m}$ (between $11 \mathrm{~m}$ and $24 \mathrm{~m}$ from the starting wall). The time spent to cover this distance was measured with a manual chronometer (Golfinho Sports MC 815, Aveiro, Portugal) by two expert evaluators and the mean value was used for further analysis.

Active drag $\left(D_{a}\right)$ was calculated as (Kolmogorov \& Duplischeva, 1992):

$$
D_{a}=\frac{D_{b} \cdot v_{b} \cdot v^{2}}{v^{3}-v_{b}^{3}}
$$

where $D_{a}$ represents the swimmer's active drag at maximal velocity, $D_{b}$ is the resistance of the perturbation buoy and, $v_{b}$ and $v$ are the swimming velocities with and without the perturbation device, respectively. 
The drag of the perturbation buoy was calculated from the manufacturer's calibration of the buoydrag characteristics and its velocity (Kolmogorov \& Duplischeva, 1992). The active drag coefficient $\left(C_{D a}\right)$ was calculated as:

$$
C_{D a}=\frac{2 \cdot D_{a}}{\rho \cdot S \cdot v^{2}}
$$

where $\rho$ is the density of the water (assumed to be $1000 \mathrm{~kg} / \mathrm{m}^{3}$ ), $D_{a}$ is the swimmer's active drag, $v$ is the swimmer's velocity and $S$ is the projected frontal surface area of the swimmers.

For the $S$ measurement, swimmers were photographed with a digital camera (DSC-T7, Sony, Tokyo, Japan) in the transverse plane from above (Caspersen, Berthelsen, Eik, Pâkozdi, \& Kjendlie, 2010). Subjects were on land, in the upright and hydrodynamic position. In this position the arms are being fully extended above the head, one hand over the other, with the fingers extended close together, and the head in neutral position. Subjects wore a regular textile swim body suit, a cap and goggles. On the camera shooting field a $0.945 \mathrm{~m}$ long calibration frame was placed next to the swimmer at shoulders level. The $S$ was measured with a photogrammetric technique from the subject's digital photo with specific area measuring software (Udruler, AVPSoft, USA). Procedures included: (i) scale calibration; (ii) manual digitization of the transverse trunk perimeter; (iii) output and recording of the $S$ value.

The power needed to overcome the drag force $\left(P_{D}\right)$ was computed as:

$$
P_{D}=D_{a} \cdot v \text {. }
$$

where $P_{D}$ is the power to overcome drag force, $D_{a}$ is the swimmer's active drag and $v$ is the swimmers velocity.

\subsection{Gliding decay velocity method}

Passive drag was assessed by applying inverse dynamics as suggested by Klauck and Daniel (1976). Swimmers were instructed to perform a maximal push-off from the wall while being fully immersed in the water, at a self-selected depth, which ranged approximately between 0.5 and $1.0 \mathrm{~m}$, which is within the depth range suggested by Vorontsov and Rumyantsev (2000) to avoid significant wave drag. So, the swimmer's self-chosen depth does not seem to significantly affect the magnitude of wave drag and therefore, the inter-subject variation on total drag force. Bouts were performed alone without any other swimmer in the same swim lane and in the nearby lanes to reduce drafting, pacing effects and bias in the drag force. Gliding was performed in the hydrodynamic position (head in neutral position, looking to the bottom of the swimming pool, legs fully extended and close together, arms fully extended at the front and with one hand over the other) with no segmental actions. Testing ended when swimmers reached the water surface and/or could not make any further horizontal displacement of their body gliding and/or started any limb action.

A speedo-meter cable (Swim speedo-meter, Swimsportec, Hildesheim, Germany) was attached to the swimmer's hip and the gliding velocity decay was acquired on-line at a sampling rate of $50 \mathrm{~Hz}$. Data were exported to a signal processing software (AcqKnowledge v. 3.5, Biopac Systems, Santa Barbara, USA) and filtered with a $3 \mathrm{~Hz}$ cut-off low-pass 4th order Butterworth filter.

The gliding mean velocity and the corresponding mean acceleration based upon the acceleration to time were calculated based on time-frame windows. The acceleration to time curve was obtained by numerical differentiation of the filtered velocity-time curve, using the 5th order centered formula as suggested elsewhere (Vilas-Boas et al., 2010):

$$
a_{i}=\frac{2 v_{i-2}-16 v_{i-1}+16 v_{i+1}-2 v_{i+2}}{24 \Delta t}
$$

where $a_{i}$ represents the hip's instantaneous acceleration, $v_{i}$ represents the hip's instantaneous velocity, and $t$ the time. Passive drag $\left(D_{p}\right)$ force was computed according to:

$$
D_{p}=\left(B M+m_{a}\right) \cdot a
$$

where $D_{p}$ represents the swimmer's passive drag, $B M$ the swimmers body mass, $m_{a}$ the swimmer's added water mass, estimated as being approximately $28 \%$ for subject with similar age (Caspersen et al., 2010), and $a$ the swimmer's acceleration. Passive drag coefficient $\left(C_{D p}\right)$ was calculated as: 


$$
C_{D p}=\frac{2 \cdot D_{p}}{\rho \cdot S \cdot v^{2}}
$$

where $\rho$ is the density of the water (assumed to be $1000 \mathrm{~kg} / \mathrm{m}^{3}$ ), $D_{p}$ is the swimmer's passive drag, $v$ is the swimmer's velocity and $S$ is the projected frontal surface area of the swimmers. The total drag force is the sum of the drag force components:

$$
D=D_{\mathrm{f}}+D_{\mathrm{p}}+D_{\mathrm{w}}
$$

$D$ is the swimmer's total drag force, $D_{\mathrm{f}}$ is the swimmer's friction drag component, $D_{\mathrm{p}}$ is the swimmer's pressure drag component, and $D_{\mathrm{w}}$ is the swimmer's wave drag component. The gliding decay velocity method yields essentially the underwater drag. As such, it does not include the wave formation component of the overall drag the swimmer has to overcome when swimming at the surface. The drag technique index (TDI) was estimated as a measure of swimming efficiency (Kjendlie \& Stallman, 2008). The TDI was computed as (Kolmogorov \& Duplischeva, 1992):

$$
T D I=\frac{D_{\mathrm{a}}}{D_{\mathrm{p}}}
$$

\subsection{Statistical procedures}

The homoscedasticity assumption was checked using the Levene test. Normality (defined as $\mathrm{Y} \cap \mathrm{N}$ $\left(\mu_{\mathrm{Y} \mid \mathrm{X} 1, \mathrm{X} 2, \ldots, \mathrm{XK}}, \sigma^{2}\right)$ was determined using the Shapiro-Wilk test. Descriptive statistics (mean and standard deviation) were calculated for all dependent variables in each gender. Percentage difference $(\Delta)$ according to gender was also reported for all dependent variables. Since the reduce sample size $(N<30)$ and the rejection of the null hypothesis $\left(\mathrm{H}_{0}\right)$ in the normality assessment, non-parametric procedures were adopted.

Mann-Whitney tests were computed to compare significant differences in the dependent variables ( $\left.d v, D_{a}, C_{D a}, P d, D_{p}, C_{D p}, T D I\right)$ according to the independent variable (boys versus girls). $Z$ values presented are based on positive ranks. Cohen's $d$ was used for all comparisons to assess the difference effect size. As a rule of thumb, it was considered a (Cohen, 1988): (i) small effect size if $0 \leqslant|d| \leqslant 0.2$; (ii) medium effect size if $0.2<|d| \leqslant 0.5$ and; (iii) large effect size if $|d|>0.5$. The level of statistical significance was set at $p \leqslant .05$.

Partial correlations between $D_{a}$ and $d v$ controlling the effect of the swimming velocities at the kinematics' mechanical method and at the velocity perturbation method data collections for each gender and for the overall sample were performed. It was considered (Ferguson, 2009) a small effect size if $0 \leqslant|r| \leqslant 0.2$; (ii) a moderate effect size if $0.2<|r| \leqslant 0.5$, and (iii) a strong effect size if $|r|>0.5$.

Whenever data were (Winter, 2008): (i) significant $(p \leqslant .05)$ with a medium/moderate or large/ strong effect size $(d>0.02 ; r>0.02)$ it was reported as being a 'meaningful difference' or 'meaningful association'; (ii) significant $(p \leqslant .05)$ with a small effect size $(d \leqslant 0.02 ; r \leqslant 0.02)$ it was reported as being a 'significant difference' or 'significant association'.

\section{Results}

There were no significant differences between genders according to biological age $(p>.05)$. The boys were taller $(p<.05)$, heavier $(p<.05)$ and chronologically older $(p<.05)$ than the girls.

Table 1 presents the descriptive statistics for the anthropometric variables measured or estimated for further assessment of the drag force, as well as the free swimming velocities obtained at the kinematics' mechanical method and at the velocity perturbation method data collections. Regarding the velocity differences between both methods, there were no significant differences and intra-class correlation was very high for boys $(p>.05$; ICC $=.96)$, girls $(p>.05$; ICC $=.86)$ and overall sample $(p>.05$; ICC $=.95$ ).

Fig. 1 presents the comparison of all dependent variables according to gender. Boys presented a meaningfully higher $d v$ than girls $(\Delta=26.57 ; Z=-2.154 ; p=.03 ; d=1.01)$. The $D a$ was meaningfully higher in boys than in girls $(\Delta=35.77 ; Z=-2.400 ; p=.02 ; d=1.34)$, although there were no 
Table 1

Mean \pm 1 SD values of the anthropometric variables and swimming velocities obtained during data collection.

\begin{tabular}{lllc}
\hline & Boys (mean $\pm 1 \mathrm{SD})$ & Girls (mean \pm 1SD) & Overall (mean \pm 1SD) \\
\hline Projected frontal surface area $\left[\mathrm{m}^{2}\right]$ & $0.826 \pm 0.185$ & $0.726 \pm 0.081$ & $0.778 \pm 0.151$ \\
Body mass + added water mass $[\mathrm{kg}]$ & $71.69 \pm 13.72$ & $60.38 \pm 7.96$ & $66.28 \pm 12.50$ \\
Velocity @ kinematics' mechanical method $[\mathrm{m} / \mathrm{s}]$ & $1.48 \pm 0.13$ & $1.29 \pm 0.07$ & $1.39 \pm 0.14$ \\
Velocity @ Perturbation velocity method $[\mathrm{m} / \mathrm{s}]$ & $1.43 \pm 0.10$ & $1.30 \pm 0.03$ & $1.36 \pm 0.09$ \\
\hline
\end{tabular}

significant differences in the $C_{D a}(\Delta=1.49 ; Z=-0.739 ; p=.49 ; d=0.04)$. There were no significant differences according to gender for both $D p(\Delta=1.57 ; Z=-0.123 ; p=.93 ; d=0.04)$ and $C_{D p}(\Delta=18.95$; $Z=-0.893 ; p=.38 ; d=0.53)$. The TDI $(\Delta=43.51 ; Z=-2.278 ; p=.02 ; d=1.45)$ and the $P d$ $(\Delta=42.85 ; Z=-2.462 ; p=.01 ; d=1.54)$ were meaningfully higher for boys than girls.

Table 2 presents the partial correlations between $D_{a}$ and $d v$. There were positive and meaningful associations between $D_{a}$ and $d v$ when controlling isolated the effect of the swim velocity in each test and the swim velocities of both tests at the same time. The only exception was the association controlling the effect of the swim velocity using the perturbation velocity method in the boys. Significant relationships between hydrodynamic and kinematic variables were found, ranging from moderate $(r=.45, p=.05)$ to strong $(r=.87, p=.01)$ associations.

\section{Discussion}

The aim of the present study was to compare the speed fluctuation and the drag force in boys and girls. $d v$ and $D_{a}$ were higher in boys than in girls. In addition, there were significant associations between both variables when controlling for the effect of swimming velocity.
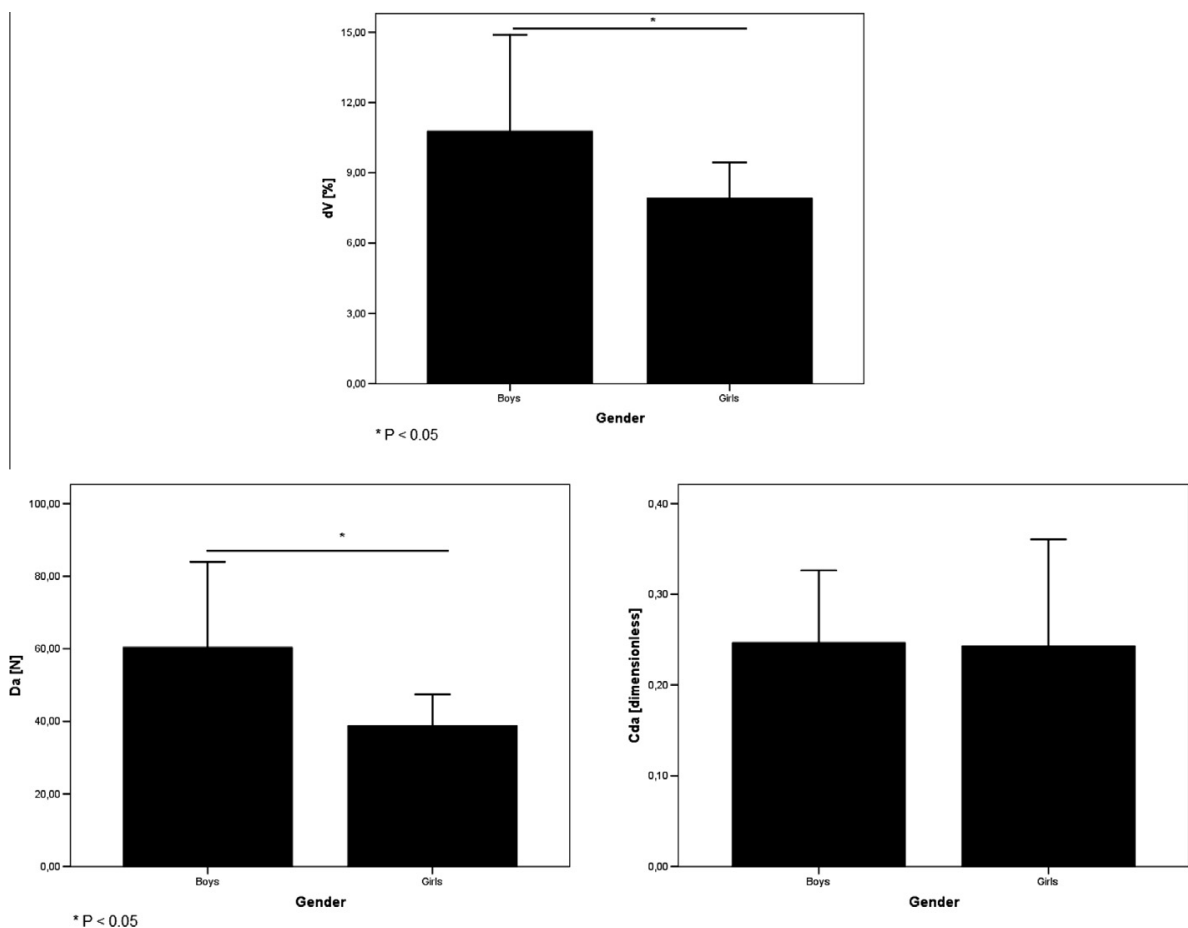

Fig. 1. Comparison of the intra-cyclic variation of the horizontal velocity of the hip $(d v)$, active drag force $\left(D_{a}\right)$, active drag coefficient $\left(C_{D a}\right)$, passive drag force $(D p)$, passive drag drag coefficient $\left(C_{D p}\right)$, power needed to overcome the drag force $\left(P_{D}\right)$ and technique drag index (TDI) between boys and girls. 

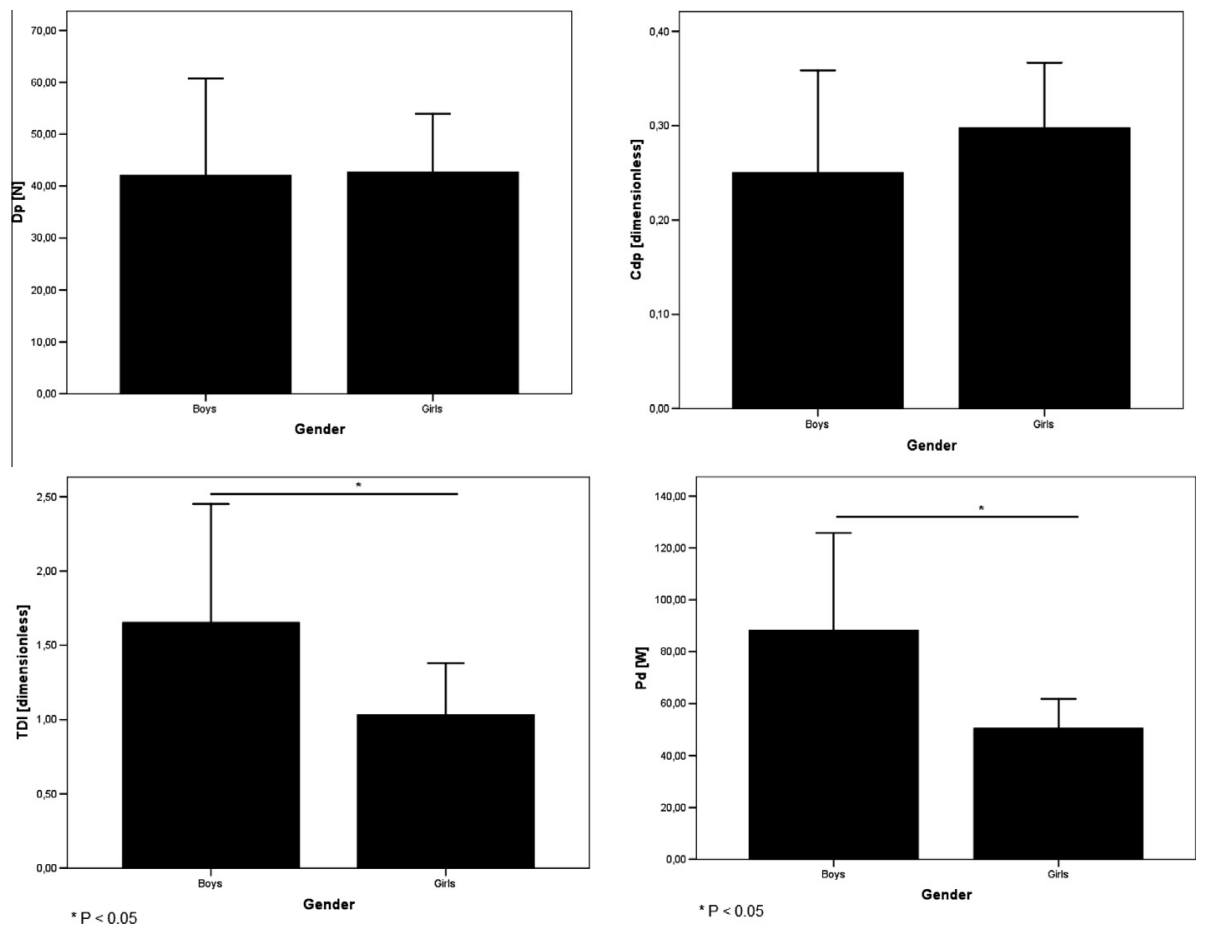

Fig. 1. (continued)

Table 2

Partial correlations between active drag ad speed fluctuation controlling the effect of the swim velocity.

\begin{tabular}{llll}
\hline & Boys & Girls & Overall \\
\hline$D_{a}$ vs $d v$ controlling Velocity @ kinematics' mechanical method + Velocity @ & 0.72 & 0.84 & 0.50 \\
Perturbation velocity method & $(\mathrm{P}=0.03)$ & $(\mathrm{P}=0.02)$ & $(\mathrm{P}=0.04)$ \\
$D_{a}$ vs $d v$ controlling Velocity @ kinematics' mechanical method & 0.70 & 0.83 & 0.51 \\
& $(\mathrm{P}=0.02)$ & $(\mathrm{P}=0.01)$ & $(\mathrm{P}=0.03)$ \\
$D_{a}$ vs $d v$ controlling Velocity @ Perturbation velocity method & 0.44 & 0.87 & 0.45 \\
& $(\mathrm{P}=0.18)$ & $(\mathrm{P}=0.01)$ & $(\mathrm{P}=0.05)$ \\
\hline
\end{tabular}

The selected anthropometric, kinematic and kinetic variables seem to present descriptive data close to other publications for the same age and/or gender (e.g., Toussaint, de Looze, van Rossem, Leijdekkers, \& Dignum, 1990). To the best of our knowledge the projected frontal surface area of young swimmers has been evaluated on a regular basis using the estimation equation of Clarys (1979) (e.g., Kjendlie \& Stallman, 2008; Marinho et al., 2010). Clarys's equation was developed using stepwise regression models that included several anthropometrical variables of sixty-three college physical education students and nine Olympic swimmers, not having a clear knowledge about model's goodof-fit to different cohort groups, including children. Clarys conducted his research in the 1970's and anthropometrical characteristics of the 70's swimmers are not the same of the XXI century ones (Morais et al., 2011). Moreover, any estimation has a small to large bias. In this sense, it was decided not to estimate the projected surface area but to actually measure it with a photometric technique popularized in the recent years (Caspersen et al., 2010; Nicolas \& Bideau, 2009; Nicolas, Bideau, Colobert, \& Berton, 2007; Vilas-Boas et al., 2010). To the best of our knowledge, there is no study regarding $d v$ in children. Comparing this research with literature focused on adult swimmers, it can be stated that 
mean values are somewhat within the lower agreement limit (1.96 SD 95\% of the interval confidence) of reported data for the same range of swim velocities in adult subjects (e.g., Barbosa et al., 2010; Seifert, Toussaint et al., 2010; Vilas-Boas et al., 2010). Kinetic variables are close (Corrêa, Alves, Botelho, Rama, \& Silva, 2007; Kolmogorov et al., 1997) or slightly higher (Kjendlie \& Stallman, 2008) than the ones reported in remaining papers for young swimmers. The bias between this research data and those reporting lower values can be related to the fact that we assessed peri-pubertal children, while the other authors assessed pre-pubertal swimmers. Peri-pubertal subjects have higher morphometric characteristics (e.g., total and partial body lengths, total and partial body mass, body areas, body volumes, etc.) than pre-pubertal subjects. There are evidences that higher morphometric characteristics lead to a higher resistance. For instance, men have higher morphometric characteristics and resistance than women (Caspersen et al., 2010; Clarys, 1979) and adults than pre-pubertal children (Kjendlie \& Stallman, 2008). So, keeping this rationality, since peri-pubertal children have higher morphometric characteristics, it is expected they might also present a higher resistance than prepubertal ones.

The velocity perturbation method takes as assumption the need of a maximal power and therefore, a maximal speed displacing with and without the hydrodynamic buoy. Since one of the aims was to understand the relationship between drag force and speed fluctuation, subject had to swim at maximal velocity in both tests. Kinematic variables, just like swimming velocity can be analyzed based on absolute (SI units, $\mathrm{m} / \mathrm{s}$ ) and relative (\%) units. For this research, gender comparison was not based on a given absolute velocity for all subjects but based on the same relative velocity (i.e., v@100\%).

The $d v, D_{a}, T D I$ and $P_{D}$ were meaningfully higher for boys than for girls. The TDI and $P_{D}$ are computed according to equations 12 and 7, respectively. So, meaningful differences according to gender in both variables are mainly explained by differences in the $D_{a}$. The $d v$ and $D_{a}$ differences among genders can be related to two main reasons: (i) kineanthropometrical factors; (ii) biomechanical factors. Re-arranging Eq. (6):

$$
D_{a}=\frac{1}{2} \cdot \rho \cdot v^{2} \cdot S \cdot C_{D a} .
$$

On regular basis it is suggested that the $S$ is constant. This must be considered just as a simplification to easily compute the passive and active drag force. To be strict during: (i) underwater gliding there is a slight positive slope for the body alignment getting to the surface at the end of the task and; (ii) stroking there are several changes in the arms, head, trunk and legs positions leading to changes in the $S$ as well. Plus, it is implicitly assumed that the passive drag component of total drag during actual swimming can be measured with the arms outstretched above the head as occurs during the passive glide test. However, during actual swimming the swimmer is: (i) at the water surface incurring wave drag as well and; (ii) the characteristic length of the swimmer will oscillate with the stroke cycle. So, some precaution should be taken into account with data interpretation.

The boys were taller and heavier than the girls, presenting a higher $S$. Plus, boys swam the bout faster than the girls. As a consequence the $D_{a}$ was higher in boys than in girls. Probably the boys also had higher muscle strength levels than the girls, leading to a higher capacity to apply propulsive force to the water. At least they were able to produce more $P_{D}$ than the girls. The $d v$ is an expression of the swimmers' acceleration. Re-arranging now equation 3 in order to the swimmer's acceleration (i.e., $d v$ ):

$$
d v=\frac{\operatorname{Pr}+D}{B M+m_{a}} .
$$

So, $d v$ is the result of propulsive and drag forces balance at a given absolute or relative velocity. Since boys presented a higher $S$ (therefore a higher $D a$ ) and a higher power (probably a higher muscle strength and propulsion), as consequence the $d v$ will increase more in the boys than in the girls. As state above boys swam faster than the females' counter partners. Probably this happened because boys were also able to apply more efficiently the propulsive forces than the girls. Propulsive efficiency can be considered as (de Groot \& van Ingen Schenau, 1988):

$$
\eta p=\frac{P_{D}}{P_{D}+P_{\text {kin }}}
$$


where $\eta p$ is the propulsive efficiency, $P_{D}$ is the power needed to overcome the drag force and $P_{k i n}$ is the power produced to transfer kinetic energy to the water mass. The question that remains is related to the partial contribution of propulsive and drag forces for the $d v$ in these young swimmers.

The gliding decay method is often cited in the literature since the seventies (Klauck \& Daniel, 1976) up to our days (e.g., Vilas-Boas et al., 2010). This means that this method is recognized as an appropriate way to assess the swimmer's passive drag. However, some limitations can be addressed. Gliding underwater does not take into account the wave drag component that the swimmer has to overcome when swimming at the surface. The $S$ is assumed to be constant, although there is a slight positive slope for the body alignment getting to the surface at the end of the gliding. A residual number of researchers suggest that during gliding, the drag force will depend on the speed squared $\left(-\mathrm{k} \mathrm{v}^{2}\right)$. This one will determine the acceleration $(a=d v / d t)$ that, in turn reduces speed; hence it should be solve a differential equation $\left(-\mathrm{kv}^{2}=\mathrm{m} \mathrm{dv} / \mathrm{dt}\right)$ for the initial speed situation.

The $D p, C_{D a}$ and $C_{D p}$ did not present significant differences in terms of gender. Naemi, Easson, and Sanders (2010) proposed a method for quantifying glide efficiency using parametric curve fitting. In this so-called 'hydro-kinematic method' the displacement over time is fitted by a parametric equation:

$$
x=C_{G} \cdot \operatorname{Ln}\left[\frac{v_{x 0}}{C_{G}} \cdot t+1\right]
$$

where $v_{x 0}$ is the initial velocity and $C_{G}$ is the glide factor calculated as:

$$
C_{G}=\frac{B M}{\frac{1}{2} \cdot S \cdot \rho} \cdot \frac{C_{m}}{C_{D p}}
$$

where $B M$ is the body mass, $S$ is the projected surface area, $\rho$ is the water density, $c_{m}$ the virtual mass coefficient and $C_{D p}$ the drag coefficient. When the curve fitting technique cannot be used, $C_{G}$ can be simplified as suggested by (Naemi \& Sanders, 2008) to:

$$
C_{G}=\frac{T}{\left(\frac{1}{v_{x}}\right)-\left(\frac{1}{v_{x 0}}\right)}
$$

where $v_{x 0}$ is the initial velocity, $v_{x}$ is the final velocity, and $T$ the duration of the gliding. In this sense, probably, although boys would be submitted to higher resistance than girls due to their morphometric characteristics, they were able to have a higher gliding efficiency as well. The higher gliding efficiency might be related once again to a higher muscle power, leading to a higher gliding velocity during more time (i.e., boys had lower gliding velocity decay).

The $d v$ characterization is a feasible way to analyze the overall swimmer's mechanics (Barbosa, Bragada et al., 2010). As is apparent from Eq. (14), $d v$ is the result of positive and negative accelerations of the body, due to its submission to propulsive and drag forces. In this sense, it can be speculated from a theoretical point of view that a positive relationship should exist between $d v$ and drag force. Increasing drag force imposes an increasing speed fluctuation. It seems that there has been only one empirical paper that dedicated some attention to this issue. Compared to adult male swimmers, the adult female swimmers had a lower $d v$, which was explained by the lower mechanical power output and the lower drag force to be overcome (Schnitzler et al., 2008). Higher drag forces would incur larger $d v$. However, since drag depends on speed squared, a comparison between swimmers is only relevant when: (i) it is made at the same absolute speed, or (ii) the speed effect is controlled in some way thereafter. Both gliding decay and velocity perturbation methods are based in the assumption that the swimmer performs the task at maximal speed (i.e., v@100\% intensity). It seems that the first option to relate the drag with $d v$ at a given range of selected absolute velocities is not suitable. Therefore, the remaining option it is to make the data collection at v@100\% and thereafter control for the effect of speed (i.e., compute partial correlations). Regarding our data, there were positive and moderate-to-strong associations between the $D_{a}$ and the $d v$ when controlling isolated the effect of the swim velocity in each test (i.e., gliding decay velocity method and perturbation velocity method) and the swim velocities of both tests at the same time in young swimmers as well. So, empirical research confirms the theoretical relationship defined for the $d v$ and the drag force. 
It can be considered as main limitations of this research: (i) the transverse trunk surface area was defined as being the projected frontal surface area of the swimmers although other authors' considered that the trunk incline plays a major role in the drag force (Zamparo et al., 2009); (ii) there is some bias assessing the hip's $d v$ instead of the centre of mass' $d v$ (Figueiredo, Vilas-Boas, Maia, Gonçalves, \& Fernandes, 2009; Psycharakis \& Sanders, 2009); (iii) for the active drag measurement there is the assumption that in both swims the power output to overcome drag is maximal and constant, but it seems there is not an agreement regarding the issue (e.g., Thorp \& Wilson, 2003; Toussaint, Roosc, \& Kolmogorov, 2004).

As a conclusion, $D_{a}$ was higher in boys than in girls of the same biological age (due to an anthropometrics and absolute swim velocity gender gap). Boys were also able to produce more $P_{D}$ than the female counterparts. As consequence, the $d v$ was higher in the boys than the girls. Added to that, there were positive associations between $D_{a}$ and $d v$ when controlling the effect of the swim velocity.

\section{Conflict of interest}

The authors have no professional relationships to disclose with companies or manufacturers who will benefit from the results of the present study.

\section{Acknowledgments}

The authors wish to thanks Jean Erik Mejias, Sérgio Jesus and Welligton Feitosa for their useful help during data collection.

\section{References}

Barbosa, T. M., Bragada, J. A., Reis, V. M., Marinho, D. A., Carvalho, C., \& Silva, A. J. (2010). Energetics and biomechanics as determining factors of swimming performance. Updating the state of the art. Journal of Science and Medicine in Sports, 13, 262-269.

Barbosa, T. M., Costa, M. J., Morais, J. E., Jesus, S., Marques, M. C., Batista, J., \& Gonçalves, J. (2011a). Conception, development and validation of a software interface to assess human's horizontal intra-cyclic velocity with a mechanical speedo-meter. In Proceedings of the XXIIIth symposium of the international society of biomechanics. Brussels.

Barbosa, T. M., Costa, M. J., Morais, J. E., Jesus, S., Silva, A. J., Batista, J., \& Gonçalves, J. (2011b). Validation with videometry of an integrated system to assess horizontal intra-cyclic velocity with a mechanical speedo-meter. In Proceedings of the XXIXth symposium of the international society of biomechanics of sports. Portuguese Journal of Sport Sciences, 11, 833-835.

Barbosa, T. M., Silva, A. J., Reis, A. M., Costa, M. J., Garrido, N., Policarpo, F., et al (2010). Kinematical changes in swimming front crawl and breaststroke with the AquaTrainer (R) snorkel. European Journal of Applied Physiology, 109, 1155-1162.

Caspersen, C., Berthelsen, P. A., Eik, M., Pâkozdi, C., \& Kjendlie, P. L. (2010). Added mass in human swimmers: Age and gender differences. Journal of Biomechanics, 43, 2369-2373.

Clarys, J. P. (1979). Human morphology and hydrodynamics. In J. Terauds \& E. W. Bedingfield (Eds.), Swimming III (pp. 3-42). Baltimore: University Park Press.

Cohen, J. (1988). Statistical power analysis for the behavioural sciences. Hillsdale, NJ: Lawrence Erlbaum Associates.

Corrêa, S. C., Alves, F., Botelho, A., Rama, L., \& Silva, A. J. (2007). Active drag and physical characteristics in age group swimmers. In H. J. Menzel \& M. H. Chagas (Eds.), Proceedings of the XXVth symposium of the international society of biomechanics of sports (pp. 204-220). Ouro Preto: Federal University of Minas Gerais.

de Groot, G., \& van Ingen Schenau, G. (1988). Fundamental mechanics applied to swimming: Technique and propelling efficiency. In B. Ungerechts, K. Wilke, \& K. Reischle (Eds.), Swimming science V (pp. 17-29). Champaign, IL: Human Kinetics Books.

di Prampero, P., Pendergast, D., Wilson, D., \& Rennie, D. (1974). Energetics of swimming in man. Journal of Applied Physiology, 37, $1-5$.

Ferguson, C. J. (2009). An effect size primer: A guide for clinicians and researchers. Professional Psychology: Research and Practice, 40, 532-538.

Figueiredo, P., Vilas-Boas, J. P., Maia, J., Gonçalves, P., \& Fernandes, R. J. (2009). Does the hip reflect the centre of mass swimming kinematics? International Journal of Sports Medicine, 30, 779-781.

Kjendlie, P. L., \& Stallman, R. K. (2008). Drag characteristics of competitive swimming children and adults. Journal of Applied Biomechanics, 24, 35-42.

Klauck, J., \& Daniel, K. (1976). Determination of man's drag coefficients and effective propelling forces in swimming by means of chronocyclography. In P. V. Komi (Ed.), Biomechanics VB (pp. 250-257). Baltimore: University Park Press.

Kolmogorov, S., \& Duplischeva, O. (1992). Active drag, useful mechanical power output and hydrodynamic force coefficient in different swimming strokes at maximal velocity. Journal of Biomechanics, 25, 311-318.

Kolmogorov, S. V., Rumyantseva, O. A., Gordon, B. J., \& Cappaert, J. M. (1997). Hydrodynamic characteristics of competitive swimmers of different genders and performance levels. Journal of Applied Biomechanics, 13, 88-97.

Malina, R. M., Bouchard, C., \& Bar-Or, O. (2004). Growth, maturation, and physical activity. Champaign, IL: Human Kinetics. 
Manley, P., \& Atha, J. (1992). Intra-stroke velocity fluctuations in paces breaststroke swimming. In D. MacLaren, T. Reilly, \& A. Lees (Eds.), Biomechanics and medicine in swimming VI (pp. 151-160). London: E \& FN Spon.

Marinho, D. A., Barbosa, T. M., Kjendlie, P. L., Vilas-Boas, J. P., Alves, F. B., Rouboa, A. I., et al (2009). Swimming simulation. In M. Peter (Ed.), Computational fluid dynamics for sport simulation (pp. 33-61). Heidelberg: Springer-Verlag.

Marinho, D. A., Barbosa, T. M., Costa, M. J., Figueiredo, C., Reis, V. M., Silva, A. J., et al (2010). Can 8-weeks of training affect active drag in young swimmers? Journal of Sports Science E Medicine, 9, 71-78.

Morais, J. E., Costa, M. J., Mejias, E. J., Marinho, D. A., Silva, A. J., \& Barbosa, T. M. (2011). Morphometric study for estimation and validation of trunk transverse surface area to assess human drag force on water. Journal of Human Kinetics, 28, 5-13.

Naemi, R., Easson, W., \& Sanders, R. H. (2010). Hydrodynamic glide efficiency in swimming. Journal of Science and Medicine in Sports, 13, 444-451.

Naemi, R., \& Sanders, R. H. A. (2008). Hydro-kinematic'method of measuring glide efficiency of a human swimmer. Journal of Biomechanical Engineering - Transactions of the Society for Mechanical Engineering, 130, 061016.

Nicolas, G., \& Bideau, B. (2009). A kinematic and dynamic comparison of surface and underwater displacement in high level monofin swimming. Human Movement Science, 28, 480-493.

Nicolas, G., Bideau, B., Colobert, B., \& Berton, E. (2007). How are Strouhal number, drag, and efficiency adjusted in high level underwater monofin-swimming? Human Movement Science, 26, 426-442.

Psycharakis, S. G., \& Sanders, R. H. (2009). Validity of the use of a fixed point for intracycle velocity calculations in swimming. Journal of Science and Medicine in Sports, 12, 262-265.

Schnitzler, C., Seifert, L., Ernwein, V., \& Chollet, D. (2008). Arm coordination adaptations assessment in swimming. International Journal of Sports Medicine, 29, 480-486.

Seifert, L., Toussaint, H. M., Schnitzler, C., Alberty, M., Chavallard, F., Lemaitre, F., et al (2008). Effect of velocity increase on arm coordination, active drag and intra-cyclic velocity variations in front crawl. In T. Nomura \& B. E. Ungerechts (Eds.), The book of the proceedings of the first international scientific conference of aquatic space activities (pp. 254-259). Tskuba: University of Tskuba.

Seifert, L., Barbosa, T. M., \& Kjendlie, P. L. (2010a). Biophysical approach to swimming: Gender effect. In S. A. Davies (Ed.), Gender gap: Causes, experiences and effects (pp. 59-80). New York: Nova Science Publishers.

Seifert, L., Toussaint, H. M., Alberty, M., Schnitzler, C., \& Chollet, D. (2010b). Arm coordination, power, and swim efficiency in national and regional front crawl swimmers. Human Movement Science, 29, 426-439.

Thorp, R., \& Wilson, B. (2003). Equal power assumption in active drag measurement. In J. C. Chatard (Ed.), Biomechanics and medicine in swimming IX (pp. 191-194). Saint-Etienne: University of Saint-Etienne.

Toussaint, H. M., de Groot, G., Savelberg, H. H., Vervoorn, K., Hollander, A. P., \& van Ingen Shenau, G. J. (1988). Active drag related to velocity in male and female swimmers. Journal of Biomechanics, 5, 435-438.

Toussaint, H. M., de Looze, M., van Rossem, B., Leijdekkers, M., \& Dignum, H. (1990). The effect of growth on drag in young swimmers. International Journal of Sport Biomechanics, 6, 18-28.

Toussaint, H. M., Roosc, P. E., \& Kolmogorov, S. (2004). The determination of drag in front crawl swimming. Journal of Biomechanics, 37, 1655-1663.

van der Vaart, A. J., Savelberg, H. H., de Groot, G., Hollander, A. P., Toussaint, H. M., \& van Ingen Schenau, G. J. (1987). An estimation of drag in front crawl swimming. Journal of Biomechanics, 20, 543-546.

Vilas-Boas, J. P., Costa, L., Fernandes, R. J., Ribeiro, J., Figueiredo, P., Marinho, D. A., et al (2010). Determination of the drag coefficient during the first and second gliding positions of the breaststroke underwater stroke. Journal of Applied Biomechanics, 26, 324-331.

Vilas-Boas, J. P., Fernandes, R. J., \& Barbosa, T. M. (2011). Intra-cycle velocity variations, swimming economy, performance and training in swimming. In L. Seifert, D. Chollet, \& I. Mujika (Eds.), Swimming: Science and performance (pp. 1191-2134). New York: Nova Science Publishers.

Winter, E. (2008). Use and misuse of the term 'significant'. Journal of Sports Sciences, 26, 429-430.

Vorontsov, A. R., \& Rumyantsev, V. A. (2000). Resistive forces in swimming. In V. M. Zatsiorsky (Ed.), Biomechanics in sport: Performance enhancement and injury prevention (pp. 184-204). Oxford: Blackwell Science.

Zamparo, P., Gatta, G., Pendergast, D., \& Capelli, C. (2009). Active and passive drag: The role of trunk incline. European Journal of Applied Physiology, 106, 195-205. 